\title{
Prevalence, Resistance Rates, and Risk Factors of Pathogens in Routine Bile Cultures Obtained during Endoscopic Retrograde Cholangiography
}

\author{
Florian P. Reiter ${ }^{a, b}$ Wolfgang Obermeier ${ }^{a, b}$ Jette Jung ${ }^{c}$ Gerald Denk $^{a, b}$ \\ Ujjwal M. Mahajan ${ }^{a, b}$ Enrico N. De Toni ${ }^{a, b}$ Jörg Schirra ${ }^{a, b} \quad$ Julia Mayerle ${ }^{a, b}$ \\ Christian Schulza, b
}

aDepartment of Medicine II, University Hospital, LMU Munich, Munich, Germany; bLiver Center, University Hospital, LMU Munich, Munich, Germany; ${ }^{C}$ Max von Pettenkofer-Institut für Hygiene und Medizinische Mikobiologie,

Ludwig-Maximilians-University Munich, Munich, Germany

\section{Keywords}

Acute cholangitis - Bile specimen - Endoscopic retrograde cholangiography

\begin{abstract}
Introduction and Objective: Acute cholangitis is a lifethreatening condition. The early initiation of antibiotic therapy significantly impacts the course of disease. Only few data are available on distribution and resistance profiles of bile pathogens. Here, we report on an analysis of routinely acquired bile specimens and provide an overview of the prevalence, resistance rates, and risk factors for the presence of pathogens in bile. Methods: Bile cultures obtained from 388 endoscopic retrograde cholangiographies (ERCs) with corresponding clinical data were analysed in 208 patients. Results: The majority (84.8\%) of cultures yielded positive for at least 1 organism. Abundance was highest for Enterococcus faecalis, Enterococcus faecium, and Escherichia coli. Multiresistant organisms were present in $14.9 \%$. The initial antibiotic regimen was changed in $44.1 \%$, which increased the length of hospital stay significantly $\left({ }^{* * *} p<\right.$ 0.001). Pre-existing papillotomy (EPT) or biliary drainage was associated with higher frequency of bile pathogens
\end{abstract}

karger@karger.com

(c) 2020 S. Karger AG, Basel

www.karger.com/ddi

Karger" $\left({ }^{* *} p<0.01\right)$ in a univariate analysis. Multivariate analysis confirmed these results for EPT and revealed significantly more positive results for pathogens, gram-negative bacteria, and fungi in patients with biliary drainage. Significant differences in the prevalence of pathogens were observed between relevant subgroups of ERC indications. The highest susceptibility rates were observed for linezolid and tigecycline in gram-positive bacteria and for meropenem and gentamicin in gram-negative bacteria. Conclusions: Our study provides a comprehensive analysis of the distribution, resistance profiles, and risk factors for the detection of bile pathogens. The frequent change in initial antibiotic treatment highlights the importance of routine bile culture and indicates that current schemas of empirical treatment might not cover the contemporary spectrum of pathogens in bile.

$$
\text { (c) } 2020 \text { S. Karger AG, Basel }
$$

\section{Introduction}

Acute cholangitis is a life-threatening condition, usually caused by obstruction of the biliary system or/and ascending pathogens due to an incompetent or resected sphincter of Oddi $[1,2]$. The early interventional restoration of the 
bile flow, mostly achieved by endoscopic retrograde cholangiography (ERC), and the early antibiotic therapy are the 2 treatment pillars in the management of acute cholangitis [3-7]. These therapeutic approaches are generally accepted and effective in clinical use. Nevertheless, standardized recommendations of antibiotic treatments are widely lacking, and the choice of the antibiotic regimens is often based on traditional centre-specific protocols. Acute cholangitis was defined by the clinical manifestations of Charcot's triad (consisting of the copresence of fever and/or chills, abdominal pain in the right upper quadrant or epigastric, and jaundice) [5]. Due to the low sensitivity of Charcot's triad for the diagnosis of acute cholangitis, new criteria were established by the Tokyo Guidelines $[8,9]$.

Acute cholangitis reflects a severe life-threatening condition, which can result in sepsis and death. Despite the fact that bile is sterile in general, asymptomatic biliary colonization was observed in patients [2], who did not show any clinical signs of infection. This condition differs from acute cholangitis. Microbiological cultures alone cannot differ between these 2 conditions in the absence of a clinical assessment. However, routine collection of bile cultures may help to detect current resistance rates and could enable or improve an early targeted therapy and may have an impact on the clinical outcome of patients. Furthermore, the knowledge of local resistance rates may enable antibiotic stewardship strategies.

Several studies investigated the distribution of pathogens in bile specimens in the past $[6,7,10-13]$. However, more contemporary studies are needed to characterize the current pathogen profile and the resistance patterns of pathogens in bile in order to optimize the therapeutic use of antibiotics in times of the increasing antibiotic resistance $[14,15]$. Due to an overuse and misuse of antibiotics, we face novel resistances [14] and decreased sensitivity to antibiotics over time. Furthermore, not only pathogens in general but also host factors such as the cause for biliary obstruction, immunodeficiency, or prior biliary interventions may reflect aspects that can influence the choice of antibiotic therapy. Unfortunately, only little evidence is available on host factors and their relevance for the choice of antibiotic therapy regimens. Therefore, new studies on advice of optimal use of antibiotics for biliary infections in modern times are warranted.

Here, we provide an overview on the prevalence and resistance rates of pathogens in bile specimens in general and in subgroups of different causes of biliary obstruction. Furthermore, we provide data on differences in patients with prior papillotomy and biliary drainages as important subgroups compared to controls.

Pathogens in Routine Bile Cultures

Obtained during ERC

\section{Materials and Methods}

This cross-sectional study was conducted at the Hospital of the University Munich, a tertiary centre. Patients were included when a bile culture for microbiological assessment was obtained during ERC between October 2016 and March 2019. During this period, bile cultures from 364 ERCs performed in 208 patients were available for analysis. In particular, bile specimens were collected routinely during this time period independently of indication and clinical suspicion of acute cholangitis, and microbiological culturing was performed. Microbiological data from bile specimens collected in clinical practice before October 2016 from patients who were included in this study according to the above-mentioned criteria were analysed if available $(n=24)$. In total, bile specimens of 388 ERCs in 208 patients were analysed in this study. Data of microbiological samples were correlated with corresponding clinical data. The analysis of patient data was achieved in a retrospective fashion.

\section{Microbiological Culturing Techniques and Antibiotic}

Susceptibility Testing

Samples were transferred to the microbiology department within $2 \mathrm{~h}$ of collection and cultured aerobically and anaerobically according to the laboratory standard procedure. Bacterial identification was performed by MALDI-TOF MS (Bruker Daltonik $\mathrm{GmbH}$, Bremen, Germany) using the manufacturer's standard protocol. Spectra were matched with the Bruker Taxonomy database version 4.0.0.2. Susceptibility testing was performed with a BD Phoenix automated AST System (Becton-Dickinson Diagnostic Systems, Heidelberg, Germany) equipped with a software for interpretation of AST results according to the EUCAST breakpoint table (document v6.0, 2017-v8.1, 2018).

\section{Endoscopic Retrograde Cholangiography}

ERC was performed using either duodenoscopes or orthograde endoscopes. The duodenoscopes used in this trial belong to the 180 er series of Olympus (TJF-Q180V; Japan). Selective cannulation of the common bile duct was indicated in all cases. Operator-dependent wire-guided cannulation with sphincterotome (e.g., double lumen sphincterotomes; Boston Scientific, Marlborough, MA, USA) or catheter (Wieser, Germany) were used in native papillae. Pretreated papillae were cannulated with balloon catheter (Olympus, Tokyo, Japan) or standard catheter with or without wire depending on operator's choice. The bile aspirates analysed in this trial were completely obtained before contrast enhancement.

ERC was performed under conscious sedation as a standard procedure using midazolam, propofol, and/or opioids according to the German guidelines [16]. The guidelines of the German Society of Digestive and Metabolic Diseases (DGVS) recommend a prophylactic antibiotic therapy prior to ERC under certain conditions [17].

\section{Ethic}

The study was approved by the ethics committee of the Medical University of Munich (18-013). The study is in line with ethical guidelines of the Declaration of Helsinki.

\section{Statistical Analysis}

Statistical calculations were performed with the SPSS 25 software package (IBM, Endicott, NY, USA) using Mann-Whitney $U$ 
Table 1. Study population. Summary of baseline characteristics, indications for endoscopic retrograde cholangiography (ERC), and relevant host factors like evidence of papillotomy, biliary drainage, or drug-induced immunosuppression

\begin{tabular}{|c|c|c|}
\hline Basis characteristics & $N$ & $\%$ \\
\hline Patients & 208 & \\
\hline ERCs & 388 & \\
\hline \multicolumn{3}{|l|}{ Gender } \\
\hline Male & 131 & 63 \\
\hline Female & 77 & 37 \\
\hline \multicolumn{3}{|l|}{ Indications } \\
\hline Cholelithiasis & 60 & 15.5 \\
\hline PSC & 34 & 8.8 \\
\hline Pancreatic carcinoma & 57 & 14.7 \\
\hline Cholangiocellular carcinoma & 40 & 10.3 \\
\hline Hepatocellular carcinoma & 8 & 2.1 \\
\hline LiTx anastomotic stricture & 55 & 14.2 \\
\hline LiTx ITBL & 33 & 8.5 \\
\hline Stent occlusion & 6 & 1.5 \\
\hline Secondary sclerosing cholangitis & 8 & 2.1 \\
\hline Others & 87 & 22.4 \\
\hline \multicolumn{3}{|l|}{ Papillotomy } \\
\hline Prior papillotomy & 312 & 80.4 \\
\hline No papillotomy & 73 & 18.8 \\
\hline Unclear & 3 & 0.8 \\
\hline \multicolumn{3}{|l|}{ Stents } \\
\hline Yes & 158 & 40.7 \\
\hline No & 201 & 51.8 \\
\hline Unclear & 29 & 7.5 \\
\hline \multicolumn{3}{|l|}{ Drug-induced immunosuppression } \\
\hline Yes & 41 & 19.7 \\
\hline No & 167 & 80.3 \\
\hline
\end{tabular}

ERC, endoscopic retrograde cholangiography; PSC, primary sclerosing cholangitis; LiTx, liver transplantation; ITBL, ischaemic type biliary lesion.

test, Pearson's $\chi^{2}$ test, and generalized estimating equations (GEE) with odds ratio, as applicable. Samples were analysed longitudinally. In the GEE analysis, pre-existing papillotomy and biliary drainage were defined with reference $=1$. $p$ values $<0.05$ were considered statistically significant. Data are presented as the means \pm standard errors of the mean.

\section{Results}

\section{Baseline Characteristics, Distribution of Pathogens,} and Choice of Initial Antibiotic Regimens

A total of $37 \%(n=77)$ of the patients in this study were female. Major indications for the intervention were cholelithiasis $(15.5 \% ; n=60)$; primary sclerosing cholangitis (PSC) $(8.8 \% ; n=34)$; ERC due to tumour obstruction
Table 2. Distribution of isolated pathogens in bile

\begin{tabular}{lrc}
\hline Pathogens & $N$ & $\begin{array}{l}\text { Positive in } \\
(\%) \text { all ERCs }\end{array}$ \\
& & \\
\hline Pathogens in bile (at least 1 pathogen) & & \\
$\quad$ Positive & 329 & 84.8 \\
$\quad$ Negative & 59 & 15.2 \\
Bacteria (10 most frequent) & & \\
Enterococcus faecalis & 129 & 33.2 \\
Enterococcus faecium & 111 & 28.6 \\
Escherichia coli & 90 & 23.2 \\
Enterobacter cloacae & 46 & 11.9 \\
Klebsiella pneumoniae & 39 & 10.1 \\
Klebsiella oxytoca & 37 & 9.5 \\
Enterobacter spp. & 34 & 8.8 \\
Streptococcus spp. & 26 & 6.7 \\
Enterococcus spp. & 26 & 6.7 \\
Pseudomonas aeruginosa & 23 & 5.9 \\
Streptococcus anginosus & 22 & 5.7 \\
Citrobacter freundii & 22 & 5.7 \\
Gram-positive (at least 1 pathogen) & 282 & 72.7 \\
Gram-negative (at least 1 pathogen) & 207 & 53.3 \\
Fungi (3 most frequent) & & \\
Candida albicans & 77 & 19.8 \\
Candida glabrata & 20 & 5.2 \\
Candida tropicalis & 10 & 2.6 \\
\hline
\end{tabular}

ERC, endoscopic retrograde cholangiography.

(34.3\%; $n=133$ ), with pancreatic carcinoma (PC), cholangiocellular carcinoma (CCC), and hepatocellular carcinoma (HCC) as leading causes for tumour obstructions $(27.1 \% ; n=105)$; or obstructive problems with or without cholangitis after liver transplantation (LiTx) $(22.7 \% ; n=$ 88). Of these patients after LiTx, ERC was indicated and performed in 55 patients due to anastomotic strictures and in 33 patients due to ischaemic type biliary lesion. The majority of ERCs were done in patients, who underwent a prior papillotomy $(80.4 \% ; n=312)$. Biliary stents were present in $40.7 \%(n=158)$ of all ERCs. $19.7 \%$ of the patients were treated with immunosuppressants after organ transplantation $(n=41)$. Baseline characteristics are given in Table 1 . The majority of specimens $(84.8 \% ; n=$ $329)$ were positive for at least 1 pathogen. $21.6 \%(n=84)$ were positive for 2 pathogens, $17.8 \%(n=69)$ for 3 pathogens, and $22.2 \%(n=86)$ were positive for 4 or more pathogens. In $23.2 \%(n=90)$, only 1 pathogen was evident.

The 10 most frequently found bacteria are listed in $\mathrm{Ta}$ ble 2 . The following 3 pathogens were detected most often in bile specimens: Enterococcus faecalis, Enterococcus faecium, and Escherichia coli.
Reiter/Obermeier/Jung/Denk/Mahajan/ De Toni/Schirra/Mayerle/Schulz 
Table 3. Distribution of isolated pathogens of 42 available positive blood cultures associated with ERC

\begin{tabular}{lll}
\hline Pathogens & $N$ & $\begin{array}{l}\text { Positive in (\%) } \\
\text { of all positive } \\
\text { blood cultures }\end{array}$ \\
\hline Bacteria (3 most frequent) & & \\
Escherichia coli & 17 & 40.5 \\
Enterococcus spp. (faecalis et faecium) & 16 & 38.1 \\
Klebsiella spp. & 10 & 23.8 \\
Gram-positive (at least 1 pathogen) & 17 & 40.5 \\
Gram-negative (at least 1 pathogen) & 31 & 73.8 \\
\hline
\end{tabular}

ERC, endoscopic retrograde cholangiography.

Table 4. Initial antibiotic regimens in our study population and the frequency of change during hospital stay

\begin{tabular}{lrr}
\hline Initial antibiotic regimens & $N$ & $\%$ \\
\hline Antibiotic regimen & & \\
$\quad$ Ceftriaxone & 48 & 23.1 \\
Piperacillin-tazobactam & 42 & 20.2 \\
Ciprofloxacin & 14 & 6.7 \\
Meropenem & 9 & 4.3 \\
Piperacillin-tazobactam + linezolid & 4 & 1.9 \\
Piperacillin-tazobactam + ciprofloxacin & 2 & 1 \\
Meropenem + linezolid & 3 & 1.4 \\
Others & 48 & 23.1 \\
Missing documentation & 1 & 0.5 \\
No anti-infective therapy & 37 & 17.8 \\
Change of antibiotic regimen & & \\
No & 90 & 52.9 \\
Yes & 75 & 44.1 \\
Missing documentation & 5 & 2.9 \\
\hline
\end{tabular}

72.7\% ( $n=282)$ of specimens yielded positive for gram-positive and $53.3 \%(n=207)$ for gram-negative bacteria. $24.2 \%(n=94)$ of all bile samples were positive for at least 1 fungus, with Candida albicans detected most often $(19.8 \% ; n=77) .61$ multiresistant bacteria were detected in 58 samples (14.9\%). 9.3\% $(n=36)$ of all cultures yielded positive for extended-spectrum beta-lactamaseproducing gram-negative Enterobacterales (ESBL), with 1 carbapenemase-positive Enterobacter cloacae; 2.8\% were positive for vancomycin-resistant Enterococcus faecium ( $n=11)$; and $2.3 \%$ were positive for linezolid-resistant pathogens $(n=9)$. Carbapenem resistance among all gram-negative was $3.1 \%$ of all cultures.

Contrary to bile samples, blood cultures were not collected routinely in this study. In 42 cases $(10.8 \%$ of all ERCs), blood cultures associated with ERC were collected

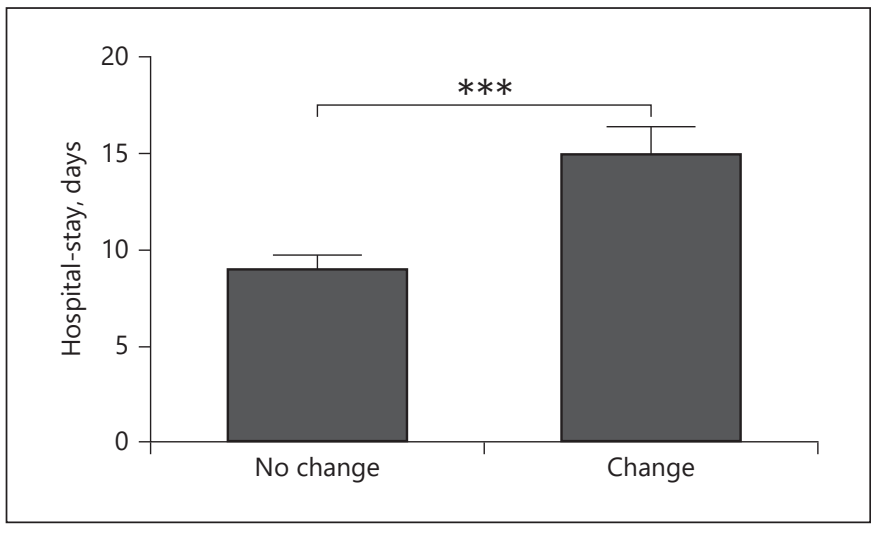

Fig. 1. Patients in whom a change of the antibiotic regimen was performed showed a longer hospital stay. The figure illustrates the duration of hospital stay (in days) in patients in whom a change or no change of antibiotic treatment was conducted $\left(n_{\text {no change }}=90 \mathrm{vs}\right.$. $n_{\text {change }}=75$; Mann-Whitney $U$ tests; $\left.{ }^{* * *} p<0.001\right)$.

and yielded positive results for pathogens. The most prevalent pathogens in blood cultures are given in Table 3. In $69.05 \%(n=29)$ of all positive blood cultures, at least 1 congruent pathogen was identified in bile cultures. Of those congruent blood-bile samples, $59.5 \%(n=25)$ had 1 congruent pathogen, $7.1 \%(n=3)$ had 2 , and 1 sample had $3(2.4 \%)$ different pathogens that were found in both specimens.

In our centre, the most common empirical treatments were ceftriaxone $(n=48)$, piperacillin-tazobactam $(n=42)$, and ciprofloxacin $(n=14)$ (Table 4$) .81 .7 \%(n=170)$ of the patients received an antibiotic therapy either therapeutically or as an antibiotic prophylaxis in the context of biliary intervention. In $44.1 \%(n=75)$ of all patients who received an antibiotic therapy at initial ERC, a change of antibiotic regimen was conducted afterwards. Patients in whom the treatment was changed showed a significant longer hospital stay $\left(9 \pm 0.7\right.$ vs. $15 \pm 1.4$ days; $n_{\text {no change }}=90$ vs. $n_{\text {change }}=$ 75; Mann-Whitney $U$ tests; ${ }^{* * *} p<0.001$ ) (Fig. 1).

In 74 cases, a clinical indication for a change of antibiotic regimen was obvious. In $56.8 \%(n=42)$ of these patients, the antibiotic therapy was changed as a consequence of the identified resistance profile of bile pathogens, and in $17.6 \%(n=13)$, the antibiotic treatment was escalated due to signs of infection or sepsis.

A change of the antibiotic regimen was conducted in $31.8 \%(n=21)$ of patients initially treated with ciprofloxacin only. Antibiotic treatment was modified in $37.9 \%$ $(n=11)$ of patients who received empirical treatment with ceftriaxone and in $42.1 \%(n=40)$ of the patients who received piperacillin-tazobactam up front. 
Table 5. Pre-existing papillotomy and biliary drainage as risk factors for detection of pathogens in bile

\begin{tabular}{|c|c|c|c|c|c|}
\hline Variable & $\begin{array}{l}\text { Evidence of } \\
\text { pathogen }(n)\end{array}$ & $\begin{array}{l}\text { No evidence of } \\
\text { pathogen }(n)\end{array}$ & $N$ & $\chi^{2}(\mathrm{df})$ & $p$ value \\
\hline \multicolumn{6}{|l|}{ Gram-positive } \\
\hline No papillotomy & 39 & 34 & 73 & $11.57(1)$ & $<0.01$ \\
\hline Pre-existing papillotomy & 101 & 31 & 132 & & \\
\hline \multicolumn{6}{|l|}{ Gram-negative } \\
\hline No papillotomy & 18 & 55 & 73 & $16.23(1)$ & $<0.001$ \\
\hline Pre-existing papillotomy & 71 & 61 & 132 & & \\
\hline \multicolumn{6}{|l|}{ Fungi } \\
\hline No papillotomy & 6 & 67 & 73 & $10.48(1)$ & $<0.01$ \\
\hline Pre-existing papillotomy & 36 & 96 & 132 & & \\
\hline \multicolumn{6}{|l|}{ Gram-positive } \\
\hline No stents & 81 & 55 & 136 & $14.80(1)$ & $<0.001$ \\
\hline Stents & 47 & 6 & 53 & & \\
\hline \multicolumn{6}{|l|}{ Gram-negative } \\
\hline No stents & 42 & 94 & 136 & $30.87(1)$ & $<0.001$ \\
\hline Stents & 40 & 13 & 53 & & \\
\hline \multicolumn{6}{|l|}{ Fungi } \\
\hline No stents & 21 & 115 & 136 & $14.75(1)$ & $<0.001$ \\
\hline Stents & 22 & 31 & 53 & & \\
\hline
\end{tabular}

Evidence of gram-positive bacteria, gram-negative bacteria, and fungi in patient after EPT or with biliary drainages was compared with patients without pre-existing EPT or without drainages $\left(\chi^{2}\right.$ test, ${ }^{* *} p<0.01$; *** $p<0.001)$.

Microbiological Pathogens Are Found More Often in Patients with Pre-Existing Papillotomy and Biliary Drainage

We next assessed if patients with pre-existing papillotomy (EPT) or biliary drainages show higher rates of positive microbiological results in bile specimens. Here, we found in a univariate analysis that after EPT or preexisting biliary drainage, microbiological assessments yielded more often positive for at least 1 gram-positive bacterium, at least 1 gram-negative bacterium, or at least 1 fungus (Table 5).

GEEs confirmed a pre-existing EPT as risk factor for the detection of pathogens, gram-positive bacteria, gramnegative bacteria, and fungi (Table 6a-d). Biliary drainages were significantly associated in the multivariate analysis with the detection of pathogens, gram-negative bacteria, and fungi (Table $6 a, c, d)$. For the detection of gram-positive bacteria, we observed a trend $(p=0.077)$ for biliary drainages as risk factor (Table 6b).

\section{Profile of Pathogens in Subgroups}

As relevant subgroups of indications for ERC, we defined cholelithiasis $(n=60)$, PSC $(n=34)$, obstructive biliary problems after LiTx (ischaemic type biliary lesion or anastomotic strictures) $(n=88)$, and ERCs due to tu- mour obstruction, with PC, CCC, and HCC as leading causes for tumour obstructions $(n=105)$. In a next step, we performed a subgroup analysis comparing these major indications for ERC in our centre using GEE.

The analysis of these most common indications for ERCs in our collective revealed significant differences in the prevalence of gram-positive bacteria, gram-negative bacteria, and fungi evident between the analysed subgroups (Table 7). Of note, in patients with PSC, grampositive bacteria were identified less frequently, gramnegative bacteria were predominately observed in patients after LiTx, and fungi were predominant in patients who received an ERC due to tumour obstructions (Table 7). These groups significantly differed from other subgroups as illustrated in Table 7.

\section{Resistance Profiles of Pathogens}

We next analysed the resistance profiles of pathogens in bile. Resistance profiles of isolated bacteria are listed in Table 8 and those of fungi in Table 9. As expected, most gram-positive bacteria were sensitive for tigecycline $\left(n_{\text {sensitive }}=256, n_{\text {all }}=263 ; 97.3 \%\right)$, linezolid $\left(n_{\text {sensitive }}=294\right.$, $\left.n_{\text {all }}=303 ; 97 \%\right)$, and vancomycin $\left(n_{\text {sensitive }}=294, n_{\text {all }}=\right.$ $330 ; 89.1 \%)$. Gram-negative bacteria were susceptible to meropenem $\left(n_{\text {sensitive }}=312, n_{\text {all }}=329 ; 94.8 \%\right)$, gentamicin
46

Dig Dis 2021;39:42-51

DOI: $10.1159 / 000509289$
Reiter/Obermeier/Jung/Denk/Mahajan/ De Toni/Schirra/Mayerle/Schulz 
Table 6. Analysis of risk factors for the positive bile cultures

\begin{tabular}{lccc}
\hline Variable & Regression coefficients & p value & Odds ratio (95\% CI) \\
\hline (a) Risk factor assessment for bile specimens that yielded positive results for pathogens & \\
Pre-existing papillotomy & 1.241 & $<\mathbf{0 . 0 1}$ & $3.365(1.632-6.941)$ \\
Biliary drainage & 0.989 & $<\mathbf{0 . 0 5}$ & $1.692(1.041-6.935)$ \\
\hline (b) Risk factor assessment for bile specimens that yielded positive results for gram-positive bacteria \\
Pre-existing papillotomy & 0.822 & $<\mathbf{0 . 0 5}$ & $2.276(1.217-4.256)$ \\
Biliary drainage & 0.526 & 0.077 & $1.682(0.945-3.031)$ \\
\hline (c) Risk factor assessment for bile specimens that yielded positive results for gram-negative bacteria \\
Pre-existing papillotomy & 0.989 & $<\mathbf{0 . 0 1}$ & $2.69(1.355-5.33)$ \\
Biliary drainage & 1.258 & $<\mathbf{0 . 0 0 1}$ & $3.519(2.033-6.091)$ \\
\hline (d) Risk factor assessment for bile specimens that yielded positive results for fungi & \\
Pre-existing papillotomy & 0.985 & $<\mathbf{0 . 0 5}$ & $2.677(1.125-6.372)$ \\
Biliary drainage & 0.842 & $<\mathbf{0 . 0 1}$ & $2.321(1.371-3.932)$ \\
\hline
\end{tabular}

By using a generalized estimating equation, we evaluated pre-existing papillotomy and biliary drainages as risk factors for detection of pathogens in bile for (a) pathogens, (b) gram-positive bacteria, (c) gram-negative bacteria, and (d) fungi ( $n=356$; GEE [generalized estimating equations], binomial logit model).

Table 7. Analysis of distribution of pathogens in relevant subgroups

\begin{tabular}{lcr}
\hline Subgroups & Mean difference & $p$ value \\
\hline Gram-positive & & $\mathbf{2 . 0 1}$ \\
Cholelithiasis versus PSC & 0.32 & $<\mathbf{0 . 0 1}$ \\
PSC versus obstructive problems after liver transplantation & -0.38 & $<\mathbf{0 . 0 5}$ \\
PSC versus other indications & -0.24 & $<\mathbf{0 . 0 5}$ \\
Tumour obstruction versus obstructive problems after liver transplantation & -0.16 & $<\mathbf{0 . 0 5}$ \\
Obstructive problems after liver transplantation versus Other indications & 0.14 & $<\mathbf{0 . 0 1}$ \\
Gram-negative & -0.28 & $<\mathbf{0 . 0 5}$ \\
Cholelithiasis versus obstructive problems after liver transplantation & -0.17 & $<\mathbf{0 . 0 5}$ \\
Cholelithiasis versus other indications & 0.16 & $<\mathbf{0 . 0 5}$ \\
Fungi & 0.21 & 0.051 \\
Cholelithiasis versus tumour obstruction & -0.014 & \\
PSC versus tumour obstruction & & \\
Tumour obstruction versus obstructive problems after liver transplantation & & \\
\hline
\end{tabular}

Evidence of gram-positive bacteria, gram-negative bacteria, and fungi was compared between the major subgroups who received an ERC in our centre $\left(n=374\right.$, generalized estimating equations; $\left.{ }^{*} p<0.05 ;{ }^{* * *} p<0.001\right)$. PSC, primary sclerosing cholangitis.

$\left(n_{\text {sensitive }}=303, n_{\text {all }}=325 ; 93.2 \%\right)$, tigecycline $\left(n_{\text {sensitive }}=\right.$ $\left.228, n_{\text {all }}=298 ; 82.2 \%\right)$, and cefepime $\left(n_{\text {sensitive }}=263\right.$, $\left.n_{\text {all }}=320 ; 82.2 \%\right)$ in $>80 \%$. The majority of gram-negative bacteria were susceptible also to ciprofloxacin $\left(n_{\text {sensitive }}=\right.$ 262, $\left.\quad n_{\text {all }}=329 ; 79.6 \%\right)$, piperacillin-tazobactam $\left(n_{\text {sensitive }}=239, n_{\text {all }}=328 ; 72.9 \%\right)$, and cefotaxime $/$ ceftriaxone $\left(n_{\text {sensitive }}=236, n_{\text {all }}=329 ; 71.7 \%\right)$. In respect of susceptibility to both gram-positive and gram-negative bacteria, only tigecycline $\left(n_{\text {sensitive }}=484, n_{\text {all }}=561 ; 86.3 \%\right.$ ) and piperacillin-tazobactam $\left(n_{\text {sensitive }}=490, n_{\text {all }}=681\right.$; $72 \%$ ) demonstrated an effectiveness with susceptibility rates higher than $70 \%$. Interestingly, the majority of all detected fungi were susceptible to the tested antifungal agents, like fluconazole $\left(n_{\text {sensitive }}=85, n_{\text {all }}=112 ; 75.9 \%\right)$, flucytosine $\left(n_{\text {sensitive }}=104, n_{\text {all }}=111 ; 93.7 \%\right)$, voriconazole $\left(n_{\text {sensitive }}=85, n_{\text {all }}=86 ; 98.8 \%\right)$, echinocandin $\left(n_{\text {sensitive }}=\right.$ $\left.104, n_{\text {all }}=111 ; 93.7 \%\right)$, or amphotericin B $\left(n_{\text {sensitive }}=20\right.$, $\left.n_{\mathrm{all}}=22 ; 90.9 \%\right)$. 
Table 8. Resistance profiles of isolated bacteria in bile

\begin{tabular}{llll}
\hline $\begin{array}{l}\text { Resistance profiles of } \\
\text { pathogens (bacteria) }\end{array}$ & $\begin{array}{l}\text { Sensitivity } \\
\text { Gram-positive, } \%\end{array}$ & $\begin{array}{l}\text { Sensitivity } \\
\text { Gram-negative, \% }\end{array}$ & $\begin{array}{l}\text { Sensitivity } \\
\text { all, \% }\end{array}$ \\
\hline $\begin{array}{l}\text { Antibiotic regimen } \\
\text { Ampicillin/amoxicillin }\end{array}$ & & & \\
Amoxicillin-clavulanic acid & 68.1 & 3.4 & 37.0 \\
Piperacillin & 70.4 & 20.9 & 46.4 \\
Piperacillin-tazobactam & 69.2 & 42.0 & 56.0 \\
Cefuroxime & 71.1 & 72.9 & 72.0 \\
Cefotaxime/Ceftriaxone & 12.3 & 45.8 & 28.4 \\
Cefepime & 12.8 & 71.7 & 41.2 \\
Ciprofloxacin & 11.9 & 80.4 & 45.8 \\
Meropenem & 2.6 & 79.6 & 42.5 \\
Gentamicin & 15.0 & 94.8 & 53.4 \\
Tigecycline & 3.2 & 93.2 & 49.1 \\
Linezolid & 97.3 & 82.2 & 86.3 \\
Vancomycin & 97.0 & $\mathrm{n} / \mathrm{a}$ & $\mathrm{n} / \mathrm{a}$ \\
\hline
\end{tabular}

\section{Discussion/Conclusion}

In light of an ever-increasing number of ERCs, current resistance profiles of bile pathogens in times of increasing antibiotic resistance are warranted $[14,15]$. Moreover, identification of host factors predisposing to bile colonization as risk factor for the development of biliary infection [18] and the resistance patterns should be taken into account. Aim of this study was to determine the prevalence of pathogens and their resistance profiles and the identification of risk factors for their presence in bile. This information could influence the choice of empirical antibiotic therapy and may have an impact on the course of acute cholangitis.

Despite a definition of acute cholangitis provided by the Tokyo Guidelines [9], we often face the problem to establish the diagnosis of acute cholangitis definitely in clinical practice. Especially in patients with tumour obstruction or in patients after LiTx, who are highly susceptible to multiple infection foci due to immunosuppression, the establishment of the diagnosis of acute cholangitis can be challenging or even not definitely possible. These situations often require a pragmatic approach in the treatment of these patients. Furthermore, a recent study [19] identified an inadequate initial antimicrobial therapy being associated with increased 30 -day mortality in patients with bacteraemic cholangitis. In our opinion, this strongly argues for a routine collection of bile culture during ERC.

In this analysis, we provide data on acquired bile specimens independently of signs of cholangitis. In this study,
Table 9. Resistance profiles of isolated fungi in bile

\begin{tabular}{lrl}
\hline $\begin{array}{l}\text { Resistance profiles of } \\
\text { pathogens (fungi) }\end{array}$ & $N$ & $\begin{array}{l}\text { Sensitivity, } \\
\%\end{array}$ \\
\hline $\begin{array}{l}\text { Antimycotic regimen } \\
\text { Fluconazole }\end{array}$ & 112 & 75.9 \\
Flucytosine & 111 & 93.7 \\
Voriconazole & 86 & 98.8 \\
Echinocandin & 111 & 93.7 \\
Amphotericin B & 22 & 90.9 \\
\hline
\end{tabular}

we included results from bile cultures that were collected during 388 ERCs in 208 patients between October 2016 and March 2019. We have to state that 388 ERCs reflect less than the estimated number of ERCs during a 2.5-year period in our centre. All colleagues who performed ERCs in our centre for this study were introduced to collect bile cultures routinely. Possible explanations for the discrepancy of estimated and included results from ERCs may be because of the fact that in some ERCs, bile cultures cannot be aspirated due to technical reasons before contrast enhancement. This fact clearly reduced the number of bile samples. If an ERC was performed shortly after first ERC, examiner may have not seen the need to collect a second bile culture. The summary of these reasons may explain the lower number of available cultures for analysis and may reflect a potential bias.

Furthermore, we cannot certainly differentiate between acute cholangitis and biliary colonizations. However, by routinely acquiring bile cultures, we can address important
48

Dig Dis 2021;39:42-51

DOI: $10.1159 / 000509289$
Reiter/Obermeier/Jung/Denk/Mahajan/

De Toni/Schirra/Mayerle/Schulz 
aspects in the treatment of acute cholangitis, a condition which is challenging to diagnose in clinical practice as mentioned above. First, we can provide an overview of distribution of pathogens and resistance profiles, which may enable antibiotic stewardship strategies in case of acute cholangitis. Second, the early analysis of bile cultures can allow or improve an early targeted therapy and may have an impact on clinical outcomes of patients.

Our analysis revealed a spectrum of microorganisms that is comparable to the results of a large multi-centre retrospective study that investigated $>6,000$ patients diagnosed with acute cholangitis in Japan and Taiwan [13]. In detail, the study by Gomi et al. [13] and our study showed that about $80 \%$ ( $83.4 \%$ [13] vs. $84.8 \%$ in this study) of all bile cultures yielded positive results. Both studies identified E. faecalis, E. faecium, E. coli, and Klebsiella pneumoniae being highly prevalent microorganisms in bile.

The microbiological results of blood cultures obtained in this study identified E. coli, Enterococcus spp., and Klebsiella spp. as the most prevalent pathogens in blood. This may indicate that all of these pathogens are of pathogenic relevance, and the results are in accordance with the distribution of microorganisms found in routinely obtained bile specimens.

In our opinion, this may underline that results from routinely acquired bile culture are comparable to the spectrum of isolates obtained from patient with acute cholangitis. Prospective studies have to address if the outcome of acute cholangitis can be improved by targeting these pathogens.

Our data illustrate that in about $44 \%$ of patients, the antibiotic regimen had to be adjusted during hospital stay. We observed that patients show a significant longer hospital stays when an adjustment of antibiotic regimen was conducted. We cannot rule out that this finding is also related to the cause or severity of underlying disease, which may prolong the hospital stay and lead to an adjustment of the antibiotic regimen independently. However, the high number suggests that standard antibiotic regimens are insufficient, and it highlights the need for more efficient regimens in the treatment of biliary infections. The reason for these high rates of adjustments may be related to the high prevalence of E. faecium (28.6\%) in bile samples, and it raises the question if initial antibiotic regimens should be considered to have activity against E. faecium. Of note and in accordance with a previous study by Rupp et al. [6], in our study, more bile culture yielded positive for gram-positive than for gram-negative bacteria. This raises the question if 3rd generation cephalosporins or ciprofloxacin, which are commonly used in therapy of biliary infections,

Pathogens in Routine Bile Cultures

Obtained during ERC reflect an adequate choice for monotherapy as neither the study by Rupp et al. [6] nor the present study revealed an adequate susceptibility of gram-positive bacteria to $3^{\text {rd }}$ generation cephalosporins or ciprofloxacin.

We have to state as limitation of this study that only patients who were treated at a tertiary centre were included. At tertiary centres, the rate of patients, who have already had a prior ERC, is high and the evaluated pathogens may not mirror profiles of all patients who undergo ERC. However, an effective initial antibiotic treatment is of paramount importance for patients in these centres, as they are often more advanced ill and less tolerant to a delay in effective treatment.

In this study, we provide resistance profiles of a broad spectrum of antibiotic substances. We found that in gram-positive bacteria, tigecycline, linezolid, and vancomycin demonstrated high susceptibility rates. In gramnegative bacteria, ciprofloxacin, piperacillin-tazobactam, and 3rd generation cephalosporins (cefotaxime/ceftriaxone) showed acceptable resistance rates. For meropenem, gentamicin, tigecycline, and cefepime, susceptibility rates were above $80 \%$. In our opinion, along with tigecycline, only piperacillin-tazobactam demonstrated acceptable effectiveness in both gram-positive and gram-negative bacteria, indicating a potential agent for an antibiotic initial regimen. It seems contradictory to this assumption when we describe in this study that $42.1 \%$ of patients, in whom a change of the antibiotic regimen was conducted, received up front piperacillin-tazobactam. One might argue that patients in whom an initial treatment with piperacillin-tazobactam was selected were more severely ill, which might have an independent influence on the adjustment of antibiotic regimens over course. We have to state here the limitation of the retrospective nature of this study, where changes or adjustments of anti-microbiological treatments were not recorded in a standardized fashion and might cause a bias in this regard.

In general, the high rates of positive microbiological results for pathogens ( 84.8 with $72.7 \%$ positive for grampositive pathogens and $53.3 \%$ positive for gram-negative pathogens) in bile and the fact that only tigecycline or piperacillin-tazobactam seems to be acceptable mono-regimes raises the question if combined antibiotic treatments that cover both the gram-positive and gram-negative spectrum should be recommended for the treatment of biliary infections.

Fungal drug resistance is a challenging topic, as the number of antifungal agents for clinical use is low and infections with more resistant Candida species are on the rise [20]. In our study, susceptibility rates for Candida 
spp. were higher for echinocandins than for fluconazole, but further data on the prevalence of Candida species in bile samples are needed to address if echinocandins should be preferred in the treatment of critically ill patients with biliary infections.

We demonstrated in this study that pre-existing EPT is a risk factor for positive microbiological testing in bile specimens. Due to the retrospective nature of our study, we cannot certainly define which patients received their "first in life" ERC. However, when we assume that patients in whom an EPT was performed during in-house intervention had their first ERC, we found E. faecium in $16.4 \%(n=12)$, which may be lower compared to the whole collective, which shows positive testing for E. faecium in $28.6 \%$. The high rate of pre-existing EPTs in our centre may be related to frequent indications for recurrent ERCs, especially seen in patients with tumour obstruction or after LiTx.

The subgroup analyses of the most common indications for ERCs in our collective revealed that there are significant differences in frequency of gram-positive bacteria, gram-negative bacteria, and fungi evident between the analysed subgroups (Table 7). This is an important finding as these host factors may influence the choice of treatment. In our opinion, further prospective studies should validate our finding that patients after LiTx show more frequently positive results for gram-negative bacteria and patients with tumour obstructions due to PC, CCC, and HCC are more often positive for fungi. Both subgroups are in general patients where an initial treatment failure may cause more severe clinical courses as these patients have generally more advanced illness. If these findings can be validated in prospective studies, protocols may imply the use of effective therapies for gram-negative bacteria or fungi in these subgroups.

Inpatient care is an important generator of healthcare costs as demonstrated in the elderly in Germany [21]. We assume that the current empirical treatment in our centre is insufficient in regard to its cost-effectiveness caused by the high rates of changes/adjustments of antibiotic therapy (44.1\% in this study). A change/adjustment of antibiotic therapy was associated with a significant longer hospital stay ( $9 \pm 0.7$ vs. $15 \pm 1.4$ ), which clearly indicates relevance for cost-effectiveness. We argue that routine analyses of resistant rates and taking these rates into account for antibiotic therapy may result in shorter hospital stays and may enable a more cost-effective treatment for patients with biliary infections.

We take the view that (a) bile culture should be routinely collected in each ERC independently of acute chol- angitis to enable early and targeted treatment and (b) further studies should also imply next-generation sequencing that shows high sensitivity for detection of microbiological pathogens [22]. In summary, our study provides a comprehensive analysis of the distribution and resistance profile in patients treated in a tertiary centre. Further prospective studies should be conducted to validate our findings to provide optimized treatment protocols for patients with acute cholangitis.

\section{Acknowledgements}

Extracts of this work were presented at the Annual Meeting of the German Society for Digestive and Metabolic Diseases (DGVS) 2019 (Z Gastroenterol 2019; 57: e183-e396; KV 274) in Wiesbaden. This paper is dedicated to the memory of our wonderful young colleague Wolfgang Obermeier, who sadly passed away recently. Wolfgang was a highly intelligent and ambitious medical student who collected and analysed the data used in this paper in the framework of his thesis. Our deepest thoughts and sympathies go out to his wife, his wonderful children, and his family. We will honour and cherish his memory with deep gratitude.

\section{Statement of Ethics}

The published research complies with the guidelines for human studies and are ethically in accordance with the World Medical Association Declaration of Helsinki. The study was approved by the ethics committee of the Medical University of Munich (18-013).

\section{Conflict of Interest Statement}

G.D. received advisory board and lecture fees and travel support from AbbVie, Falk Foundation, Gilead, GMP Orphan, Intercept, and Novartis. The other authors declare that they have no conflicts of interest.

\section{Funding Sources}

No funding was necessary for this study.

\section{Author Contributions}

F.P.R., W.O., and C.S. act as the submission's guarantors. F.P.R., W.O., and C.S. collected the data. F.P.R., W.O., U.M.M., and C.S. analysed the data. F.P.R., W.O., and C.S. designed the study and wrote the paper. J.J., G.D., U.M.M., E.N.T., J.S., and J.M. contributed to the design of the study and interpreted the data. All authors approved the final version of the article, including the author list.
Reiter/Obermeier/Jung/Denk/Mahajan/

De Toni/Schirra/Mayerle/Schulz 


\section{References}

1 Sung JY, Costerton JW, Shaffer EA. Defense system in the biliary tract against bacterial infection. Dig Dis Sci. 1992;37(5):689-96.

2 Sokal A, Sauvanet A, Fantin B, de Lastours V. Acute cholangitis: diagnosis and management. J Visc Surg. 2019;156(6):515-25.

3 Devière J, Motte S, Dumonceau JM, Serruys E, Thys JP, Cremer M. Septicemia after endoscopic retrograde cholangiopancreatography. Endoscopy. 1990;22(2):72-5.

4 Lai EC, Mok FP, Tan ES, Lo CM, Fan ST, You $\mathrm{KT}$, et al. Endoscopic biliary drainage for severe acute cholangitis. N Engl J Med. 1992; 326(24):1582-6.

5 Wada K, Takada T, Kawarada Y, Nimura Y, Miura F, Yoshida M, et al. Diagnostic criteria and severity assessment of acute cholangitis: Tokyo Guidelines. J Hepatobiliary Pancreat Surg. 2007;14(1):52-8.

6 Rupp C, Bode K, Weiss KH, Rudolph G, Bergemann J, Kloeters-Plachky P, et al. Microbiological assessment of bile and corresponding antibiotic treatment: a strobe-compliant observational study of 1401 endoscopic retrograde cholangiographies. Medicine. 2016;95(10):e2390.

7 Negm AA, Schott A, Vonberg RP, Weismueller TJ, Schneider AS, Kubicka S, et al. Routine bile collection for microbiological analysis during cholangiography and its impact on the management of cholangitis. Gastrointest Endosc. 2010;72(2):284-91.

8 Kiriyama S, Takada T, Strasberg SM, Solomkin JS, Mayumi T, Pitt HA, et al. New diagnostic criteria and severity assessment of acute cholangitis in revised Tokyo Guidelines. J Hepatobiliary Pancreat Sci. 2012;19(5):54856.

9 Yokoe M, Hata J, Takada T, Strasberg SM, Asbun HJ, Wakabayashi G, et al. Tokyo Guidelines 2018 diagnostic criteria and severity grading of acute cholecystitis (with videos). J Hepatobiliary Pancreat Sci. 2018;25: $17-30$.

10 Lorenz R, Herrmann M, Kassem AM, Lehn N, Neuhaus H, Classen M. Microbiological examinations and in-vitro testing of different antibiotics in therapeutic endoscopy of the biliary system. Endoscopy. 1998;30(8):70812.

11 Ehrenstein BP, Salamon L, Linde HJ, Messmann H, Schölmerich J, Glück T. Clinical determinants for the recovery of fungal and mezlocillin-resistant pathogens from bile specimens. Clin Infect Dis. 2002;34(7):902-8.

12 Leung JW, Ling TK, Chan RC, Cheung SW, Lai CW, Sung JJ, et al. Antibiotics, biliary sepsis, and bile duct stones. Gastrointest Endosc. 1994;40(6):716-21.

13 Gomi H, Takada T, Hwang TL, Akazawa K, Mori R, Endo I, et al. Updated comprehensive epidemiology, microbiology, and outcomes among patients with acute cholangitis. J Hepatobiliary Pancreat Sci. 2017;24(6):310-8.

14 Ventola CL. The antibiotic resistance crisis: part 1: causes and threats. P T. 2015;40(4): 277-83.

15 Ventola CL. The antibiotic resistance crisis: part 2: management strategies and new agents. P T. 2015;40(5):344-52.
16 Riphaus A, Wehrmann T, Hausmann J, Weber B, von Delius S, Jung M, et al. [S3-guidelines "sedation in gastrointestinal endoscopy" 2014 (AWMF register no. 021/014)]. Z Gastroenterol. 2015;53(8):802-42.

17 Denzer U, Beilenhoff U, Eickhoff A, Faiss S, Hüttl P, In der Smitten S, et al. [S2k guideline: quality requirements for gastrointestinal endoscopy, AWMF registry no. 021-022]. Z Gastroenterol. 2015;53(12):E1-227.

18 Kiesslich R, Holfelder M, Will D, Hahn M, Nafe $\mathrm{B}$, Genitsariotis R, et al. [Interventional ERCP in patients with cholestasis. Degree of biliary bacterial colonization and antibiotic resistance]. Z Gastroenterol. 2001;39(12):985-92.

19 Tagashira Y, Sakamoto N, Isogai T, Hikone M, Kosaka A, Chino R, et al. Impact of inadequate initial antimicrobial therapy on mortality in patients with bacteraemic cholangitis: a retrospective cohort study. Clin Microbiol Infect. 2017;23(10):740-7.

20 Cowen LE. The evolution of fungal drug resistance: modulating the trajectory from genotype to phenotype. Nat Rev Microbiol. 2008; 6(3):187-98.

21 Heider D, Matschinger H, Müller H, Saum KU, Quinzler R, Haefeli WE, et al. Health care costs in the elderly in Germany: an analysis applying Andersen's behavioral model of health care utilization. BMC Health Serv Res. 2014; $14: 71$.

22 Amann RI, Ludwig W, Schleifer KH. Phylogenetic identification and in situ detection of individual microbial cells without cultivation. Microbiol Rev. 1995;59(1):143-69. 